\title{
A Neural Mechanism of Strategic Social Choice under Sanction-Induced Norm Compliance ${ }^{1,2,3}$
}

\author{
Aidan Makwana, ${ }^{1}$ Georg Grön, ${ }^{2}$ Ernst Fehr, ${ }^{1}$ and Todd A. Hare ${ }^{1}$
}

DOI:http://dx.doi.org/10.1523/ENEURO.0066-14.2015

${ }^{1}$ Laboratory for Social and Neural Systems Research, Department of Economics, University of Zurich, 8006 Zürich, Switzerland, and ${ }^{2}$ University of Ulm, University Hospital for Psychiatry, 89075 Ulm, Germany

\begin{abstract}
In recent years, much has been learned about the representation of subjective value in simple, nonstrategic choices. However, a large fraction of our daily decisions are embedded in social interactions in which value guided decisions require balancing benefits for self against consequences imposed by others in response to our choices. Yet, despite their ubiquity, much less is known about how value computation takes place in strategic social contexts that include the possibility of retribution for norm violations. Here, we used functional magnetic resonance imaging (fMRI) to show that when human subjects face such a context connectivity increases between the temporoparietal junction (TPJ), implicated in the representation of other peoples' thoughts and intentions, and regions of ventromedial prefrontal cortex (VmPFC) that are associated with value computation. In contrast, we find no increase in connectivity between these regions in social nonstrategic cases where decision-makers are immune from retributive monetary punishments from a human partner. Moreover, there was also no increase in TPJ-vmPFC connectivity when the potential punishment was performed by a computer programmed to punish fairness norm violations in the same manner as a human would. Thus, TPJ-vmPFC connectivity is not simply a function of the social or norm enforcing nature of the decision, but rather occurs specifically in situations where subjects make decisions in a social context and strategically consider putative consequences imposed by others.
\end{abstract}

Key words: decision-making; fMRl; functional connectivity; norm compliance; strategy

\section{Significance Statement}

A large fraction of our decisions are embedded in social contexts that require balancing benefits for self against the positive or negative reactions of others in response to our choices. Yet, how the brain computes the value for different courses of action in such choices is unknown. We examined the neurobiological mechanisms underlying strategic social choices in the context of potential retributive punishment. Our findings indicate that there are specific increases in the functional interactions between brain regions previously associated with mentalizing about others' beliefs and key nodes of the brain's value computation system during choices in which it is necessary to balance direct personal gains against the likelihood of subsequent norm enforcing punishment by other people.

\section{Introduction}

A large portion of our daily decisions are embedded in social interactions in which the values of different behaviors depend on the behavior of relevant others. Such interactions range from major decisions about whether to

Received November 24, 2014; accepted May 28, 2015; First published June 16, 2015.

${ }^{1}$ The authors report no conflict of interest. apply for a new job and risk upsetting current colleagues to mundane choices about how much to tip the bartender

\footnotetext{
${ }^{2}$ Author contributions: This work represents a novel set of analyses on an existing dataset. G.G. and E.F. designed and performed the original research; A.M. and T.A.H. designed and conducted the current analyses; A.M., G.G., E.F., and T.A.H. wrote the paper.

${ }^{3}$ This work was supported in part by the Swiss National Science Foundation Grant 140277 to T.A.H. and the NCCR in Affective Sciences to E.F.
} 
at your preferred pub in order to maintain favored patron status. In these and many other situations, social norm compliance must be considered to avoid peer punishment for norm violations. In all these cases, we need to take the likely reactions of other people into account. However, despite their ubiquity, very little is known about how value computation takes place in contexts where one's own behavior may trigger subsequent responses that affect subjective values.

As a central component of the brain's decision circuitry, the ventromedial prefrontal cortex (vmPFC) has been associated with value computation in nonstrategic decision contexts ranging from primary to social rewards for both self and others (Nicolle et al., 2012; Bartra et al., 2013; Clithero and Rangel, 2013) and in choices during competitive games (Hampton et al., 2008; Zhu et al., 2012). In addition, vmPFC lesions have been shown to alter choice behavior under strategic conditions where norm violations can result in retributive punishment (Krajbich et al., 2009). Collectively, these data suggest that vmPFC might compute subjective values in strategic social choices that require balancing personal preferences with predictions about how the reactions of others to norm violations will impact outcomes for self, but this idea has not yet been directly tested. Furthermore, how predictions about the opponents' behavior enter into vmPFC value computations is unknown. One hypothesis is that such information is provided to VmPFC by regions that are involved in mentalizing about others.

Previous research has shown that inferring another person's beliefs in order to estimate his probable future actions recruits neural circuits including the temporoparietal junction (TPJ; Saxe and Wexler, 2005; Frith and Frith, 2006; Zhu et al., 2012). Moreover, studies on competitive and cooperative interpersonal games suggest that TPJ encodes information about other players that could be used to guide choices (Behrens et al., 2008; Hampton et al., 2008; Coricelli and Nagel, 2009; Bhatt et al., 2010; Hare et al., 2010; Rilling and Sanfey, 2011; Carter et al., 2012; Morishima et al., 2012; Carter and Huettel, 2013). However, whether information encoded in TPJ is incorporated into vmPFC value signals during social norm enforcement choices is unknown. Therefore, we sought to examine whether TPJ-vmPFC interactions underlie value computations in this type of strategic social choice.

We examined brain activity using functional magnetic resonance imaging (fMRI) during decisions about the division of monetary assets between participants paired with either another human (social treatment) or a computer partner programmed to enforce social norm violations (nonsocial treatment). On each trial, participants had to choose how to divide 100 monetary units between

Correspondence should be addressed to Todd A. Hare, Laboratory for Social and Neural Systems Research, Department of Economics, University of Zurich, Blümlisalpstrasse 10, 8006 Zürich. E-mail: todd.hare@econ.uzh.ch. DOI:http://dx.doi.org/10.1523/ENEURO.0066-14.2015

Copyright (C) 2015 Makwana et al.

This is an open-access article distributed under the terms of the Creative Commons Attribution 4.0 International, which permits unrestricted use, distribution and reproduction in any medium provided that the original work is properly attributed. themselves and the partner. However, these monetary allocation decisions were made in two distinct contexts. In the punishment context, the partner could punish perceived violations of the social norm for fairness by paying to reduce the participant's earnings, whereas in the control condition the partner could not enforce norm compliance through retributive punishment. The combination of these treatments and conditions allowed us to examine brain activity that was specific to choices that were both social and required strategic reasoning to optimize direct monetary gain against the probability of profit-reducing punishments for fairness norm violations.

\section{Materials and Methods}

\section{Participants}

Forty-seven healthy, right-handed male students performed a strategic economic game while undergoing fMRI scanning. Participants were screened for fMRI contraindications including acute medical conditions and psychiatric or neurological illness. All participants provided written informed consent in accordance with the local ethics committee.

\section{Behavioral Paradigm}

The behavioral paradigm proceeded as follows. On each trial, participants split 100 monetary units (MUs) between themselves (Player A) and Player B. For 24 participants Player B represented a human counterpart (social treatment group, mean age $\pm \mathrm{SD}, 23.5 \pm 2.3$ years) and for 23 participants Player B was a computer (nonsocial treatment group, mean age \pm SD, $24.8 \pm 1.9$ years). Participants were randomly assigned to either the social or nonsocial treatment groups upon arrival for the experiment. One participant from the social treatment was excluded from all analyses for a lack of comprehension of the task and two participants from the nonsocial group were excluded from the fMRI analyses described below because they never transferred any MUs (leaving 23 social and 21 nonsocial participants). The social group was instructed that each human Player B's punishment decisions had been acquired in a previous experiment using the strategy method. This method involved Player B making a decision about how many monetary units to spend on punishment if Player A transferred a specific amount. The punishment rate selected by human Player Bs decreased with greater transfers in an approximately linear fashion. The data from all Player Bs was used to generate a punishment distribution function and program the computer algorithm for the nonsocial treatment. The nonsocial group participants were instructed that they were playing against a computer that had been programmed to simulate the responses of the previous human Player B group and were given the same details as the social treatment participants about the strategy method of choice elicitation for Player Bs. All participants were randomly matched against different players on each trial (i.e. a one-shot game). Payment included 20 Euros for participating and 1 Euro per $100 \mathrm{MU}$ earned. Each trial consisted of a treatment screen indicating the trial type for $6 \mathrm{~s}$, a participant driven decision period (mean $4.3 \mathrm{~s}$, SD $2.7 \mathrm{~s}$ ), then a wait 
period of $6 \mathrm{~s}$ followed by a feedback screen displayed for $6 \mathrm{~s}$. Trials were separated by a fixation cross ITI for $6-8.7$ $\mathrm{s}$, sampled from a uniform distribution, thus the decision period started at least $12 \mathrm{~s}$ after the previous trials feedback. During the task, participants faced 12 control trials (CON) and 12 punishment trials (PUN) in a random order as indicated during the treatment screen. In CON trials, Player B was not able to punish Player A for making a selfish split (i.e. a dictator game scenario); however, in PUN trials Player B could punish Player A by 5 MUs for each $1 \mathrm{MU}$ spent. Both participants began every trial with a reserve of $25 \mathrm{MUs}$ and therefore, Player B was always able to punish Player A completely (i.e. take away all earnings) during the punishment trials.

\section{Behavioral Analysis}

The behavioral variable of interest was the amount kept/transferred by participants in the role of Player $A$ as a function of group and condition. There was a nonnormal distribution of transferred amounts in CON trials (Kolmogorov-Smirnoff test, $p=0.03$ ), therefore, we analyzed the transfer amount data using nonparametric Wilcoxon signed rank (paired) and Kruskal-Wallis rank sum tests. All $p$ values reported are based on two-sided tests. To better describe the punishment distributions, we linearly regressed punishment on the transfer amount for the social and nonsocial groups.

\section{MRI Acquisition}

Blood oxygen level-dependent (BOLD) echo planar imaging (EPI) scans were performed on a 3 Tesla Siemens Magnetom Allegra using 32 slices and a voxel resolution of $2 \times 2 \times 2 \mathrm{~mm}(+0.5 \mathrm{~mm}$ slice gap), with a TR of 2490 $\mathrm{ms}$, and a TE of $38 \mathrm{~ms}$. All fMRI data was acquired during a single scanning session (mean length of $750 \mathrm{~s}, \mathrm{SD} 38.5$ s). A full brain EPI (56 slices using the same parameters as functional EPI) and anatomical scan (sagittal MPRAGE T1 sequence with a voxel size of $1 \times 1 \times 1 \mathrm{~mm}$ ) were also acquired. The fMRI data preprocessing included slicetime correction, spatial realignment to the mean EPI image for each subject, normalization to MNI space, and smoothing with a $10 \mathrm{~mm}$ FWHM Gaussian kernel using the SPM 8 software (Wellcome Department of Imaging Neuroscience, Institute of Neurology, London, UK).

\section{fMRI Analysis}

Our primary GLM (GLM-1) was computed to examine BOLD activity relating to the amount kept/transferred during the decision period. GLM-1 modeled four regressor types: (1) treatment, (2) decision, (3) wait, and (4) feedback periods in all trials (PUN and CON) and separately for CON only (8 regressor onsets in total). Single $0 \mathrm{~s}$ duration stick functions were convolved with the canonical HRF for the treatment, decision and feedback periods, and a $6 \mathrm{~s}$ boxcar function was used for convolution during the wait period. In addition, we used three parametric regressors (PR): (PR1) kept amount at decision onset in all trials, (PR2) kept amount at previous within-condition decision, and (PR3) profit amount at feedback onset for all trials. Six motion parameter regressors were also included in GLM-1. Note that the initial endowment is fixed at 100
MUs for every trial, and therefore, a positive correlation with the amount kept by Player A (PR1) implies a negative correlation with amount transferred to Player B.

SPM 8 software was used to estimate GLM-1 and compute contrasts of interest in each individual participant.

At the second level, we used the "randomise" function from the FSL 5.0.6 software package (http://www.fmrib.ox.ac.uk/fsl/) to test for regions that reflected the amount kept across all participants. We computed a one sample t-test on the single participant contrasts for positive correlations with the kept amount regressor together with a nuisance variable ( $0=$ social, $1=$ nonsocial $)$ to explain variance due to social and nonsocial participant groups. We performed the $t$ test using the nonparametric permutation algorithm in randomise in combination with the threshold-free cluster enhancement (TFCE) method implemented in FSL (Smith and Nichols, 2009). Test statistics and $p$ values were derived from 5000 permutations. We corrected for multiple comparisons using familywise error correction at the whole-brain level to achieve corrected significance levels of $p<0.05$.

\section{PPI Analysis}

For each participant, a seed time course in vmPFC was extracted from a $4 \mathrm{~mm}$ sphere centered on the voxel with the strongest correlation with kept amount in that participant from within the overlapping voxels for the group vmPFC cluster generated by GLM-1 and an anatomical mask of vmPFC including the rectal gyrus, medial orbitofrontal, and anterior cingulate cortex below $z=5$ (5464 8 $\mathrm{mm}^{3}$ voxels) based on the AAL atlas (Tzourio-Mazoyer et al., 2002). The vmPFC time series was deconvolved as outlined by Gitelman et al., (2003) before creating the psychophysiological interaction regressors. For the psychophysiological interaction (PPI) GLM (GLM-PPI), the vmPFC time series was used as a physiological regressor and interacted with two separate psychological boxcar regressors for the decision period in CON and PUN conditions. This resulted in two separate psychophysiological interaction terms in GLM-PPI. In GLM-PPI, the decision period duration was modeled as $5 \mathrm{~s}$ before the first button press. This expanded window was used because the precise timing of the amount to keep/transfer computation within the treatment and decision screen periods cannot be determined in this task. However, this timing resolution limitation would not bias the results in favor of any specific decision type and, if anything, works against the current findings by adding noise to the analysis. GLMPPI consisted of the following nine regressors: (1) vmPFC time series, (2) CON decision period boxcar, (3) PUN decision period boxcar, (4) CON decision $\times$ vmPFC, (5) PUN decision $\times$ vmPFC, (6) CON wait period ( $6 \mathrm{~s}$ boxcar), (7) PUN wait period (6 s boxcar), (8) CON profit screen (6 $s$ boxcar), and (9) PUN profit screen ( $6 \mathrm{~s}$ boxcar). Note that, a one-way ANOVA for the SDs of the PPI regressors for group and condition showed that they were not significantly different $\left(F_{(1,83)}=1.14, p=0.338\right)$ suggesting that the PPI analysis was not biased against CON conditions where kept amounts showed less variance. Similar 
Table 1 Regions correlating with the amount kept by Player A at the time of choice

\begin{tabular}{|c|c|c|c|c|c|c|}
\hline Region & Hemisphere & Extent & $x$ & $y$ & $z$ & Peak T \\
\hline Lingual gyrus & $\mathrm{R} / \mathrm{L}$ & 1257 & 8 & -74 & 8 & 5.21 \\
\hline Cingulate gyrus & $\mathrm{R} / \mathrm{L}$ & 39 & 2 & -10 & 36 & 4.54 \\
\hline vmPFCa-ACC & $\mathrm{R} / \mathrm{L}$ & 36 & 0 & 48 & -2 & 4.14 \\
\hline mPFC-paracingulate gyrus & $\mathrm{R} / \mathrm{L}$ & 30 & 0 & 54 & 4 & 3.79 \\
\hline mPFC-ACC & $\mathrm{R} / \mathrm{L}$ & 28 & -4 & 44 & 14 & 3.91 \\
\hline Frontopolar cortex/IFG & $\mathrm{R}$ & 23 & 42 & 44 & 0 & 4.75 \\
\hline dmPFCb-paracingulate/SFG & $\mathrm{R} / \mathrm{L}$ & 21 & -2 & 50 & 26 & 5.45 \\
\hline Occipital cortex & $\mathrm{R}$ & 20 & 42 & -76 & -6 & 4.69 \\
\hline $\mathrm{ACC}$ & $\mathrm{R} / \mathrm{L}$ & 18 & 0 & 26 & 28 & 4.22 \\
\hline Thalamus & L & 16 & -12 & -34 & 8 & 3.90 \\
\hline vmPFC-ACC & $\mathrm{R}$ & 14 & 8 & 48 & 0 & 3.82 \\
\hline Frontopolar cortex & L & 11 & -16 & 58 & 28 & 5.00 \\
\hline Cingulate gyrus & L & 10 & -4 & -4 & 32 & 3.95 \\
\hline
\end{tabular}

Peak coordinates $(x, y, z)$ are listed in MNI space. T values are test statistics derived from 5000 permutations of the data. All regions are significant at $p<0.05$ whole-brain familywise error corrected for multiple comparisons.

IFG, Inferior frontal gyrus; SFG, superior frontal gyrus; R, right; L, left.

${ }^{a}$ vmPFC cluster used as a mask to extract subject specific time courses for PPI analyses.

${ }^{b} \mathrm{dmPFC}$ cluster used as a mask to extract subject specific time courses for PPI analyses.

to GLM-1, parametric regressors for kept amount at decision, previous kept amount at decision and profit amount at feedback were included for both punishment and control conditions. Last, GLM-PPI included the six motion parameter regressors. A PPI analysis using the dorsomedial prefrontal cortex (dmPFC) seed noted in Table 1 was also performed. The analysis was identical to GLM-PPI, except that the BOLD time courses were extracted from the dmPFC ROI rather than the vmPFC ROI described above.

Following estimation of GLM-PPI in SPM8, single participant contrasts were computed for regressors of interest. At the second level, we again used TFCE and the nonparametric permutation function, randomise, to test for between group differences in connectivity with vmPFC. Test statistics and $p$ values were derived from 5000 permutations. Based on previous work (Morishima et al., 2012) showing that social preferences during interpersonal interactions are linked to structural and functional differences in the TPJ, we created a spherical ROI with $10 \mathrm{~mm}$ radius around the $\mathrm{MNI}$ coordinates $(x, y, z=$ $60,-44,18)$. The conjunction of this $\mathrm{ROI}$ and the group functional coverage mask was used for small volume correction (324 $8 \mathrm{~mm}^{3}$ voxels). This functional coverage map was utilized because the acquisition parameters for the functional MRI data did not provide whole-brain coverage, and in some cases, the tilt of the transverse slices relative to anterior commissure-posterior commissure resulted in lack of coverage for the superior temporal and inferior parietal cortex. Forty-two participants $(21$ social and 21 nonsocial) had adequate functional coverage and were included in the PPI analysis. We corrected for multiple comparisons using familywise error correction within this mask to achieve small volume correction (SVC) of $p<$ 0.05 .

The bar plots shown in Figure $3 c$ were created by taking the average vmPFC- TPJ PPI coefficients from all voxels in the functional ROI for the difference between social and nonsocial punishment trials shown in Figure $3 a$. These bar plots are presented for visualization purposes only and were not used as a basis for any statistical analysis.

In addition to comparing the PPIs during the PUN decisions between groups, we also tested for an association between the vmPFC-TPJ PPI during punishment decisions and the average punishment received by each individual within the social and nonsocial groups. We applied the same TPJ small volume correction described above for this analysis.

Last, we performed a post hoc analysis of correlations with profit during the PUN feedback condition (GLM-PPI regressor 9) by extracting PUN profit betas from all significant voxels in the social PUN PPI cluster shown in Figure $3 b$.

\section{Results}

Behaviorally, there was no difference in the total amounts transferred between the social and nonsocial treatment groups (Kruskal-Wallis $\mathrm{X}_{(1, N=88)}^{2}=0.48, p=$ 0.49). Transfers in the social CON condition were on average 9.3 MU (SD 17.0), leading to an average percentage split of $22.9 \%$ for Player B after accounting for the 25 MU reserve amount for both players. These transfer rates are consistent with average rates $(\sim 20 \%)$ reported in the previous literature (Camerer, 2003). Participants in the role of Player A transferred more in PUN than CON conditions in both the social [Wilcoxon signed rank $(\mathrm{W})=276, p=$ $2.88 \mathrm{e}-5]$ and the nonsocial treatments $(\mathrm{W}=231, p=$ 6.36e-5; Fig. 1). These results suggest that Player $A$ strategically increased the amount transferred to Player B to decrease the likelihood that Player B would exercise his punishment option and reduce Player A's earnings regardless of whether Player B was a human or a computer programmed to mimic human reactions. Increasing the amount transferred in PUN trials was in fact the best strategy for Player $A$ to maximize his earnings because the punishment amount decreased with greater transfers (with zero punishment above a transfer of $50 \mathrm{MUs}$ ) in an approximately linear fashion (Fig. 2). 


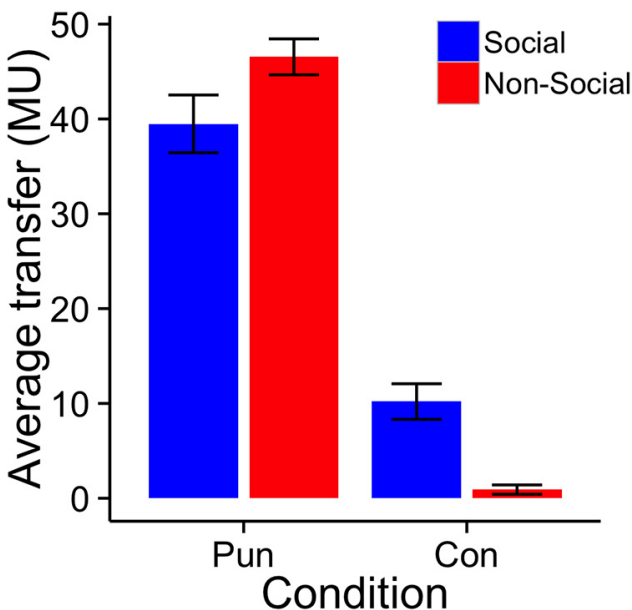

Figure 1 Amounts transferred by Player $A$ in the PUN and the CON condition of both the social and the nonsocial treatment. Transfers are represented in experimental monetary units out of a given amount of 100 units. Error bars represent the standard error of the mean for the group mean. Paired sample Wilcoxon signed rank tests (social $\mathrm{W}=276, p=2.88 \mathrm{e}-5$; nonsocial $\mathrm{W}=$ $231, p=6.36 \mathrm{e}-5$ ) showed significant differences between the PUN and CON transfer rates in each group.

In our initial neuroimaging analysis, we examined the degree to which vmPFC activity reflected value computations during monetary transfer decisions in both treatment types using a general linear model on BOLD signals. This analysis showed a positive association between kept amounts and VmPFC BOLD activity (Fig. 3a; $p<0.05$ whole-brain corrected) across all participants. In addition

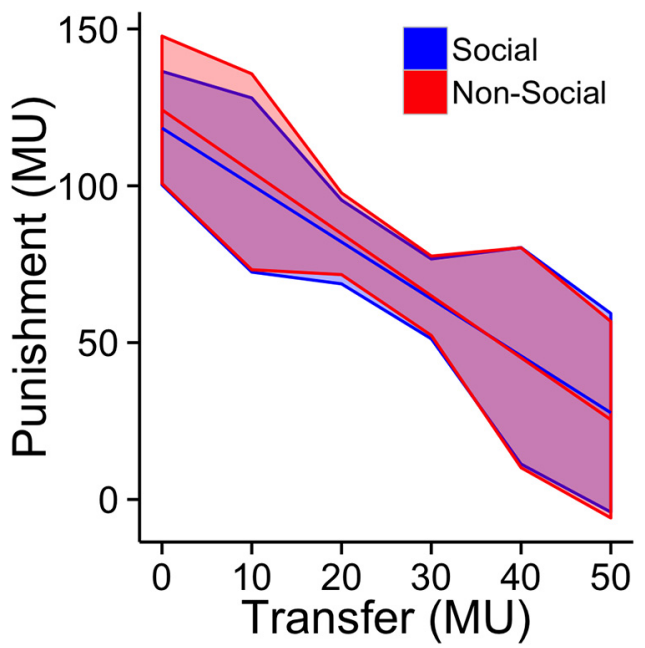

Figure 2 The plot shows punishment distributions as a function of amount transferred for both social (blue) and nonsocial groups (red). Punishment was regressed onto transfers up to $50 \mathrm{MUs}$, with the predicted punishment (thick line) and the SDs of the residuals (shaded area) for each transfer amount. Transfers $>50$ MU resulted in zero punishment. The overlapping distributions for the social and nonsocial treatments indicate that the computer algorithm was successful in replicating human punishment behavior. to $\mathrm{VmPFC}$, BOLD activity in dmPFC, right frontopolar cortex, and occipital regions also correlated with the amount kept at the time of choice (Table 1). The correlation between amount kept and BOLD activity in the vmPFC ROI was not significantly different between treatment groups (two-sample $t$ test, $t_{(42)}=1.4, p=0.332$ uncorrected) indicating that participants playing against humans and computers represented the amount kept to an equal degree in vmPFC. Furthermore, there was no significant difference between the social and nonsocial groups in the correlation with amount kept and BOLD activity in any brain region after correcting for multiple comparisons.

The vmPFC result is consistent with theoretical models and existing empirical data suggesting a central role for vmPFC in the computation of subjective values for a wide range of decision contexts (Kable and Glimcher, 2009; Rangel and Hare, 2010; Rushworth et al., 2012; Bartra et al., 2013; Clithero and Rangel, 2013). Such theories also posit that if vmPFC acts as a general valuation system, then its interactions will be modulated such that coupling with regions providing decision relevant information will increase.

Next, we tested the hypothesis that the coupling between $\mathrm{VmPFC}$ and the right TPJ will increase more during decisions that require strategic evaluations of another person's response to the outcome than in complexity matched control conditions using a PPI analysis with the vmPFC as the seed region. This analysis examines whether the correlations between vmPFC activity and other brain regions differ in social versus nonsocial PUN transfer decisions. Note that in both the social and nonsocial PUN conditions participants need to make strategic transfer decisions that take into account Player B's likely level of punishment (i.e. fairness norm enforcement), and it is only the nature of Player B (human vs computer) that differs between groups. We found that participants in the social treatment showed more positive correlations between TPJ and vmPFC in PUN trials compared with the nonsocial treatment (Fig 3; $p<0.05$ SVC; peak $\mathrm{T}=3.97$ at $x, y, z=60,-48,16$; extent $=115$ voxels). Post hoc one-sample $t$ tests showed that the average PPI effect in these voxels for social PUN was greater than zero $\left(t_{(20)}=\right.$ 2.51; $p=0.021$ ), whereas the average PPI effect for nonsocial PUN was less than zero $\left(t_{(20)}=-3.79 ; p=\right.$ $0.001)$. Exploratory analyses revealed no other regions that showed this pattern of connectivity with vmPFC after correcting for multiple comparisons. However, for completeness, we also list regions exceeding a threshold determined by the lowest individual voxel $t$ statistic $(t>$ 2.29) derived from the right TPJ cluster (Table 2). Furthermore, there were no voxels that showed a significant PPI effect in either social or nonsocial CON trials after correcting for multiple comparisons within the independent TPJ ROI or in the entire volume.

To test whether vmPFC-TPJ PUN PPI strength is related to the overall strategic play of the participants, we tested whether the individual PPI difference contrast (PUN - CON) differentially correlated with participants' average punishment amounts in the social compared with nonso- 

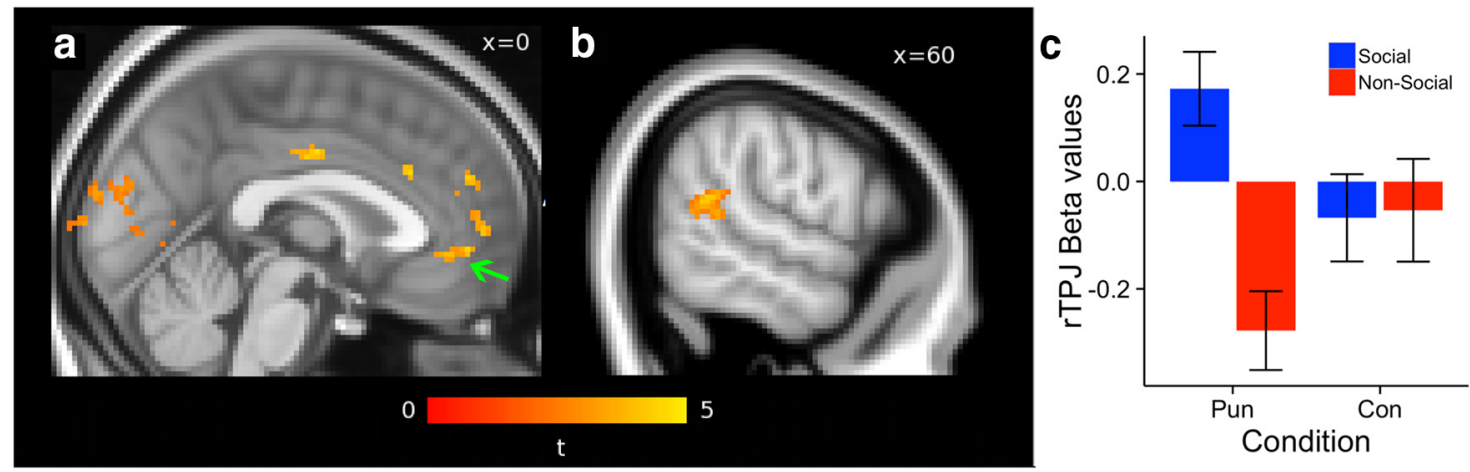

Figure 3 Activity and connectivity at the time of choice. a, Regions showing a positive correlation with the amount of monetary units participants decided to keep on each trial across all decision types. The green arrow indicates the vmPFC cluster used to extract time courses for the PPI analysis. $\boldsymbol{b}$, Region of TPJ showing increased functional connectivity with vmPFC during strategic decisions made in social punishment compared with nonsocial punishment contexts. All voxels shown in $\boldsymbol{a}$ and $\boldsymbol{b}$ are significant at $p<0.05$ after correcting for multiple comparisons. $\boldsymbol{c}$, Bar graph showing the relative coupling between vmPFC and TPJ by treatment group and choice context and demonstrating that increased TPJ-vmPFC coupling is specific to choices that are both strategic and social in nature. Error bars represent the standard error of the mean for the group mean. These bar plots are presented for visualization purposes only and were not used as a basis for any statistical analysis.

cial groups. This second level, between subjects regression analysis revealed a link between vmPFC-TPJ PPI during PUN trials and average punishment levels that were stronger in social more than nonsocial treatment participants. In the social group, greater vmPFC-TPJ PPI was associated with less punishment by Player B, whereas there was no significant relationship in the nonsocial group (Fig. 4; $p<0.05$ SVC; peak $\mathrm{T}=3.88$ at $x, y$, $z=56,-50,16$; extent 8 voxels).

For completeness, we repeated our PPI analysis replacing the VmPFC seed with a region of dmPFC that also correlated with amount kept at the time of choice. We tested this dmPFC seed in addition to VmPFC because the dmPFC has been implicated in alternative value representation and strategic mentalizing processes (Frith and Frith, 2006; Hampton et al., 2008; Coricelli and Nagel, 2009; Nicolle et al., 2012; Zhu et al., 2012). However, we found no significant differences in connectivity with the dmPFC during social compared with nonsocial PUN trials within our TPJ ROI or at the whole-brain level after correcting for multiple comparisons.

We also examined brain activity at the time of outcome when subjects learned how much profit they had made in the previous trial. We found that the parametric regressor for profit magnitude at outcome (PR3 from GLM-1) correlated with BOLD activity in several regions, including bilateral striatum and left lateral frontal cortex $(p<0.05$ whole-brain corrected; Table 3). Just as with the BOLD correlations at the time of choice, there were no regions showing a difference between the social and nonsocial groups in the correlation with profit magnitude at outcome. In addition, we conducted an ROI analysis on the BOLD correlation with profit at feedback using the voxels from the TPJ cluster showing the PUN PPI difference between the groups. We found that across all subjects there was a significantly negative effect of profit on TPJ activity at the time of feedback (one sample $t_{(41)}=-2.15$, $p=0.037$ ), and once again, the groups did not significantly differ in this effect (two sample $t_{(40)}=0.13, p=$ 0.90).

\section{Discussion}

Our results indicate a role for vmPFC in the computation of value during strategic choices involving norm enforcement and suggest that increased TPJ-vmPFC coupling is especially important in decisions that involve strategic

Table 2 Location and extent of functional clusters showing a difference in PPI with vmPFC between social and nonsocial PUN decisions that was greater than or equal to the effect in our a priori TPJ region

\begin{tabular}{llllcc}
\hline Region & Hemisphere & Extent & $x$ & $y$ & $z$ \\
TPJ & $\mathrm{R}$ & 144 & 60 & -48 & 16 \\
Parahippocampal gyrus & $\mathrm{R}$ & 93 & 22 & -26 & -14 \\
Lingual gyrus & $\mathrm{R}$ & 86 & 28 & -50 & 3.97 \\
Fusiform cortex & $\mathrm{L}$ & 78 & -34 & -38 & 3.87 \\
Fusiform cortex & $\mathrm{L}$ & 66 & -38 & -2 & -18 \\
White matter/insular cortex & $\mathrm{L}$ & 60 & -28 & -16 & -32 \\
STG & $\mathrm{L}$ & 51 & -52 & -24 & 4.12 \\
& & & & & 3.31 \\
\hline
\end{tabular}

Peak coordinates $(x, y, z)$ are listed in MNI space. T values are test statistics derived from 5000 permutations of the data. Clusters reported are all of those that surpass a threshold set by lowest $t$ value in the small volume corrected TPJ cluster $(t>2.29)$ and minimum cluster size of 50 voxels $(2 \times 2 \times 2$ mm). Note that these results are reported here for completeness only and are not corrected for multiple comparisons and thus not the subject of any inference in this paper. STG, Superior temporal gyrus. 

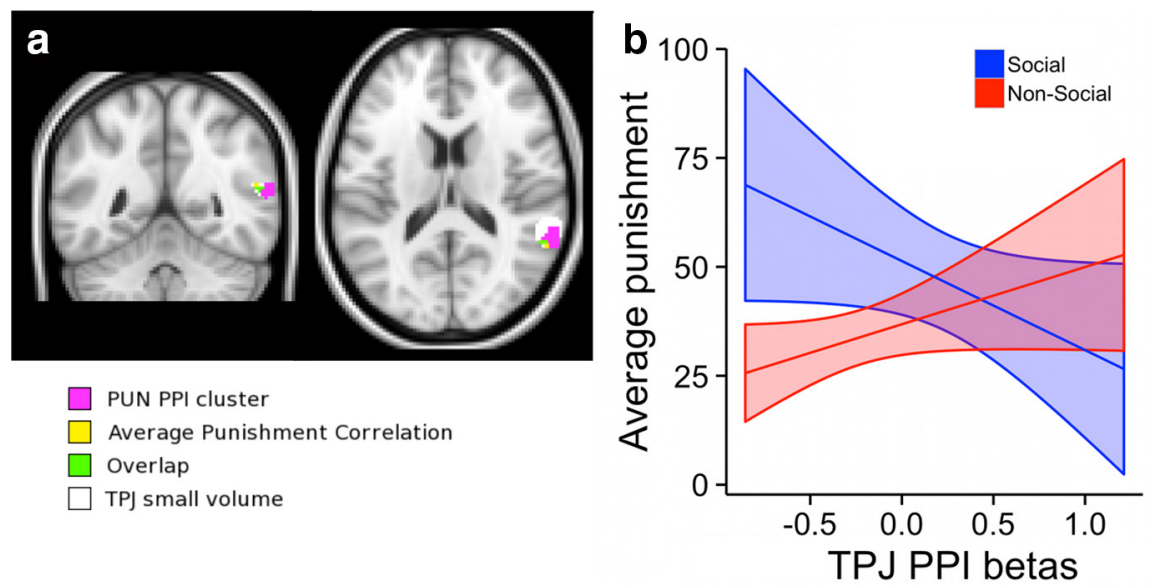

Figure 4 Regions of the TPJ relating to the vmPFC PPI at time of choice. $\boldsymbol{a}$, The voxels in magenta show greater increases in connectivity with vmPFC during PUN choices in the social compared with the nonsocial group, controlling for connectivity in CON trials. Voxels in yellow are those where the PUN - CON PPI was significantly correlated with individual average punishment levels in the social, but not the nonsocial group. Green voxels represent the overlap of both effects. Clusters are significant at $p<0.05$ SVC within the TPJ small volume shown in white. $\boldsymbol{b}$, The fitted regression slopes between TPJ-vmPFC PPI at the time of choice and an individual's average punishment level separately for the social (blue) and nonsocial (red) groups. The shading around the regression lines indicates the $95 \%$ confidence intervals.

considerations of how social others will react to one's own actions. Despite the fact that participants were fully informed that the computer opponents were programmed to punish fairness norm violations at the same levels as real human players, the coupling between TPJ and vmPFC value computation regions did not increase in nonsocial PUN decisions, and in fact, decreased relative to the nondecision baseline.

This pattern of TPJ results is consistent with previous experiments showing that multivariate analyses of TPJ activity could be used to help predict bet and fold decisions in a simplified poker game against human opponents, but including TPJ activity measures actually decreased the model's predictive power for computer opponents (Carter et al., 2012). These previous experiments did not however examine the connectivity between TPJ and other brain regions. Our TPJ-vmPFC connectivity results demonstrate that in the realm of value-based choices, TPJ-vmPFC coupling increases during strategic choices when paired with human counterparts, but decreases with computer partners. Moreover, increased connectivity between VmPFC and TPJ at the time of

Table 3 Regions correlating with profit at the time of feedback

\begin{tabular}{lllllll}
\hline Region & Hemisphere & Extent & $x$ & $y$ & $z$ & Peak T \\
Insula/striatum & $\mathrm{R} / \mathrm{L}$ & 1645 & 32 & 12 & 4 & 6.97 \\
Striatum & $\mathrm{L}$ & 475 & -30 & -14 & 10 & 5.5 \\
Frontopolar cortex & $\mathrm{L}$ & 325 & -38 & 60 & 4 & 4.88 \\
Precentral gyrus & $\mathrm{R}$ & 44 & 58 & -4 & 22 & 4.17 \\
Caudate tail & $\mathrm{R}$ & 16 & 18 & -4 & 26 & 3.85 \\
Posterior insula & $\mathrm{L}$ & 16 & -38 & -18 & 0 & 4.32 \\
Parietal operculum & $\mathrm{L}$ & 10 & -48 & -30 & 22 & 4.28
\end{tabular}

Peak coordinates $(x, y, z)$ are listed in MNI space. T values are test statistics derived from 5000 permutations of the data. All regions are significant at $p$ $<0.05$ whole-brain familywise error corrected for multiple comparisons. choice is associated with more advantageous strategic decision-making (i.e. lower norm-enforcing punishment) in social but not nonsocial contexts. This is consistent with the idea that vmPFC incorporates information from distributed brain regions into value computations and that inputs are either enhanced or inhibited as a function of their relevance in the current state. Moreover, TPJvmPFC coupling did not significantly increase in either the social or the nonsocial CON trials where punishment predictions were not necessary because the opponent could not respond. This indicates that TPJ-vmPFC connectivity was not simply a function of the social nature of the decision, but rather occurred selectively when both social and strategic factors were in play.

Previous work has shown that TPJ activity reflects social learning signals in the context of repeated interactions where it is advantageous to learn about other human players (Behrens et al., 2008; Hampton et al., 2008). This learning takes the form of update signals measuring deviations from the expected result at the time of feedback when decision outcomes are shown. These update or error signals are presumably used to guide subsequent choices when paired with the same person in the future, although the impact of TPJ activity at the time of subsequent choices was not explicitly examined in these previous reports. In the current paradigm, participants are paired with a different human partner on each trial, and therefore, outcomes of previous trial choices cannot be directly applied to future decisions. However, it may be that TPJ activity also plays a role in forming expectations based on average or normative behavior. There is a strong social norm for fairness and this norm could be used as a basis for predicting the degree of punishment by an unknown Player B that would result from various monetary splits. Consistent with this role, we found that TPJ activity increased when participants were shown feedback indi- 
cating that a strategic adjustment was necessary (i.e. low profits) on the following choice to avoid future norm enforcing responses from Player B. Moreover, the results summarized in Figure 4 suggest that increased connectivity between TPJ and VmPFC may be a mechanism by which such predictions are incorporated into value computations at the time of choice.

In addition to vmPFC, BOLD activity in several other brain regions, particularly dmPFC, correlated with the amount kept for oneself when deciding how to allocate MUs on each trial. The correlation with kept amount in dmPFC is of particular interest given previous findings that activity in this region relates to individual differences in type or level of reasoning during social interactions (Hampton et al., 2008; Coricelli and Nagel, 2009; Zhu et al., 2012). Our findings in dmPFC build on these previous individual difference results and demonstrate that this region also reflects choice specific components of strategic valuation during decisions in which social norm compliance can be enforced through peer punishment. Although the current dataset was not designed to distinguish between the value related activity in regions such as vmPFC and dmPFC, previous reports have suggested that there is a dorsal to ventral gradient for modeled and executed value functions along the mPFC (Nicolle et al., 2012). If our subjects are engaging in predictive forecasting (i.e. modeling) of Player B's responses to their transfers and decisions are taken (i.e. executed) on the basis of these models, then this could explain why we find activity correlated with the amount kept in both ventral and dorsal portions of mPFC. However, further experiments will be necessary to test this speculative hypothesis.

One limitation of the current dataset is that there were a relatively small number of choices for each participant per condition $(n=12)$. Therefore, it is possible that future studies including more choices per participant, and thus having greater power, will find additional changes in vmPFC connectivity associated with social strategic decision-making.

Decisions that balance welfare for self with the impacts on and reactions of others to one's own choices are ubiquitous in social life. Our results provide insights into the neural mechanisms underlying such behavior and suggest a key role for interactions between TPJ and vmPFC. These findings are an important advance in our understanding of the neurobiology underlying strategic social choice and provide a basis for future investigations into this central aspect of human behavior.

\section{References}

Bartra O, McGuire JT, Kable JW (2013) The valuation system: a coordinate-based meta-analysis of BOLD fMRI experiments examining neural correlates of subjective value. Neuroimage 76:412427. CrossRef Medline

Behrens TE, Hunt LT, Woolrich MW, Rushworth MF (2008) Associative learning of social value. Nature 456:245-249. CrossRef Medline

Bhatt MA, Lohrenz T, Camerer CF, Montague PR (2010) Neural signatures of strategic types in a two-person bargaining game. Proc Natl Acad Sci U S A 107:19720-19725. CrossRef Medline
Camerer C (2003) Behavioral game theory: experiments in strategic interaction. Princeton, NJ: Princeton UP.

Carter RM, Huettel SA (2013) A nexus model of the temporal-parietal junction. Trends Cogn Sci 17:328-336. [PMC] [10.1016/ j.tics.2013.05.007] [23790322]

Carter RM, Bowling DL, Reeck C, Huettel SA (2012) A distinct role of the temporal-parietal junction in predicting socially guided decisions. Science 337:109-111. CrossRef Medline

Clithero JA, Rangel A (2013) Informatic parcellation of the network involved in the computation of subjective value. Soc Cogn Affect Neurosci 9:1289-1302.

Coricelli G, Nagel R (2009) Neural correlates of depth of strategic reasoning in medial prefrontal cortex. Proc Natl Acad Sci U S A 106:9163-9168. CrossRef Medline

Frith CD, Frith $U$ (2006) How we predict what other people are going to do. Brain Res 1079:36-46. CrossRef Medline

Gitelman DR, Penny WD, Ashburner J, Friston KJ (2003) Modeling regional and psychophysiologic interactions in fMRI: the importance of hemodynamic deconvolution. Neuroimage 19:200-207. Medline

Hampton AN, Bossaerts P, O'Doherty JP (2008) Neural correlates of mentalizing-related computations during strategic interactions in humans. Proc Natl Acad Sci U S A 105:6741-6746. CrossRef Medline

Hare TA, Camerer CF, Knoepfle DT, Rangel A (2010) Value computations in ventral medial prefrontal cortex during charitable decision making incorporate input from regions involved in social cognition. J Neurosci 30:583-590. CrossRef Medline

Kable JW, Glimcher PW (2009) The neurobiology of decision: consensus and controversy. Neuron 63:733-745. CrossRef Medline

Krajbich I, Adolphs R, Tranel D, Denburg NL, Camerer CF (2009) Economic games quantify diminished sense of guilt in patients with damage to the prefrontal cortex. J Neurosci 29:2188-2192. CrossRef Medline

Morishima Y, Schunk D, Bruhin A, Ruff CC, Fehr E (2012) Linking brain structure and activation in temporoparietal junction to explain the neurobiology of human altruism. Neuron 75:73-79. CrossRef Medline

Nicolle A, Klein-Flügge MC, Hunt LT, Vlaev I, Dolan RJ, Behrens TE (2012) An agent independent axis for executed and modeled choice in medial prefrontal cortex. Neuron 75:1114-1121. CrossRef Medline

Rangel A, Hare T (2010) Neural computations associated with goaldirected choice. Curr Opin Neurobiol 20:262-270. CrossRef Medline

Rilling JK, Sanfey AG (2011) The neuroscience of social decisionmaking. Annu Rev Psychol 62:23-48. CrossRef Medline

Rushworth MF, Kolling N, Sallet J, Mars RB (2012) Valuation and decision-making in frontal cortex: one or many serial or parallel systems? Curr Opin Neurobiol 22:946-955. CrossRef

Saxe R, Wexler A (2005) Making sense of another mind: the role of the right temporo-parietal junction. Neuropsychologia 43:13911399. CrossRef Medline

Smith SN, Nichols TE (2009) Threshold-free cluster enhancement: addressing problems of smoothing-threshold dependence and localisation in cluster inference. Neuroimage 44:83-98. CrossRef Medline

Tzourio-Mazoyer N, Landeau B, Papathanassiou D, Crivello F, Etard O, Delcroix N, Mazoyer B, Joliot M (2002) Automated anatomical labeling of activations in SPM using a macroscopic anatomical parcellation of the MNI MRI single-subject brain. Neuroimage 15:273-289. CrossRef Medline

Zhu L, Mathewson KE, Hsu M (2012) Dissociable neural representations of reinforcement and belief prediction errors underlie strategic learning. Proc Natl Acad Sci U S A 109:1419-1424. CrossRef Medline 